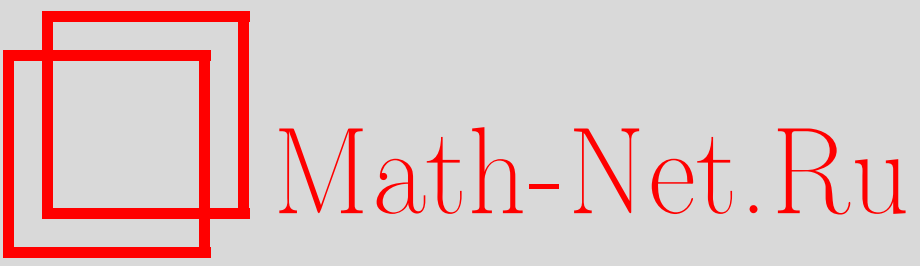

Ю. Б. Черняков, Интегрируемые системы, полученные слиянием точек из рациональной и эллиптической систем Годена, ТМФ, 2004, том 141, номер 1, 38-59

DOI: https://doi.org/10.4213/tmf111

Использование Общероссийского математического портала Math-Net.Ru подразумевает, что вы прочитали и согласны с пользовательским соглашением

http://www . mathnet.ru/rus/agreement

Параметры загрузки:

IP: 35.173 .137 .237

26 апреля 2023 г., 16:47:44 
ТЕОРЕТИЧЕСКАЯ

И МАТЕМАТИЧЕСКАЯ

ФИЗИКА

Том 141, № 1

октябрь, 2004

(C) 2004 г.

Ю. Б. Черняков*

\section{ИНТЕГРИРУЕМЫЕ СИСТЕМЫ, ПОЛУЧЕННЫЕ СЛИЯНИЕМ ТОЧЕК ИЗ РАЦИОНАЛЬНОЙ И ЭЛЛИПТИЧЕСКОЙ СИСТЕМ ГОДЕНА}

С использованием процедуры слияния отмеченных точек получены новые интегрируемые системы с полюсами порядка выше первого в матрице оператора Лакса, рассмотрены гамильтонианы этих систем, описана симплектическая структура и симметрии. С применением предела Иноземцева к полученной системе в эллиптическом случае найдена система типа Тоды с нетривиальными коммутационными соотношениями между переменными фазового пространства.

Ключевые слова: интегрируемые системы, системы Хитчина, оператор Лакса, рациональная и эллиптическая системы Годена, предел Иноземцева, слияние точек.

\section{1. ВВЕДЕНИЕ}

В работах [1]-[4] изучались системы, являюшиеся примерами систем Хитчина [5], т.е. интегрируемыми системами на кокасательных расслоениях к пространствам модулей голоморфных расслоений над кривыми. Эти системы могут быть получены симплектической редукцией [4], [5] исходного фазового пространства. Важный способ описания интегрируемых систем - рассмотрение оператора Лакса, совпадаюшего с решением уравнения отображения момента при проведении симплектической редукции. Разложение степеней оператора Лакса по подходящему базису дает гамильтонианы интегрируемой системы. В вышеуказанных работах изучались операторы Лакса, допускаюшие полюсы первого порядка в отмеченных точках.

В настоящей работе мы попытаемся ответить на следующие естественно возникающие вопросы. Как выглядят системы с полюсами более высокого порядка в операторе Лакса? Какова их симплектическая форма? Как устроено пространство модулей этих систем?

Здесь используются гамильтонианы, операторы Лакса и симплектическая структура рацииональной и эллиптической систем Годена. Их краткое описание будет дано в разделе 2.

${ }^{*}$ Институт теоретической и экспериментальной физики, Москва, Россия. E-mail: chernyakov@gate.itep.ru 
Результаты слияния точек в случае рациональной системы Годена. В рациональном случае гамильтонианы системы (раздел 3 ), полученной при слиянии двух (из $n$ отмеченных) точек $\left(x_{b} \rightarrow x_{a}\right)$, имеют вид

$$
\begin{gathered}
H_{1}^{2,2}=\left\langle\left(p^{0}\right)^{2}\right\rangle+2 \sum_{c \neq a, b} \frac{\left\langle p^{1} p_{c}\right\rangle}{x_{a}-x_{c}}, \quad H_{1}^{2,1}=\sum_{c \neq a, b}^{n}\left[\frac{\left\langle p^{0} p_{c}\right\rangle}{x_{a}-x_{c}}-\frac{\left\langle p^{1} p_{c}\right\rangle}{\left(x_{a}-x_{c}\right)^{2}}\right], \\
H_{c}^{2,1}=2 \frac{\left\langle p^{1} p_{c}\right\rangle}{\left(x_{c}-x_{a}\right)^{2}}+\frac{\left\langle p^{0} p_{c}\right\rangle}{x_{c}-x_{a}}+\sum_{d \neq c, a, b} \frac{\left\langle p_{c} p_{d}\right\rangle}{x_{c}-x_{d}},
\end{gathered}
$$

где $\langle\ldots\rangle$ означает след. Скобки Ли-Пуассона между переменными $p_{a}$ определены формулой (7), а между переменными $p^{r}$ - формулой (22) (см. ниже). Операторы Лакса этих систем были описаны в работе [6]. Отметим, что при слиянии $r$ точек из рассматриваемых $n$ оператор Лакса имеет вид

$$
L_{\text {new }}=\frac{p^{r} k_{r}}{\left(z-x_{a}\right)^{r}}+\cdots+\frac{p^{1} k_{1}}{\left(z-x_{a}\right)^{2}}+\frac{p^{0} k_{0}}{z-x_{a}}+\sum_{c \neq a, b} \frac{p_{c}}{z-x_{c}}
$$

где $k_{r}$ - некоторые коэффициенты, зависящие от параметров разложения $p^{r}$, параметров слияния точек и некоторых условий, возникающих из требования отсутствия сингулярностей в конечном выражении. Элементам $p^{r}$ соответствуют верхнетреугольные матрицы, образующие параболическую алгебру, изоморфную алгебре многочленов от $\varepsilon^{-1}$ с коэффициентами $p^{r}$. Коммутационные соотношения между элементами $p^{r}$ будут указаны в таблице 1 . Отметим, что специальный случай оператора Лакса (2) был получен в работе [7].

Результаты слияния точек в случае эллиптической системы Годена. В эллиптическом случае (раздел 4) мы получили следуюшие выражения для гамильтонианов новой системы при слиянии двух точек $(p \in \operatorname{sl}(2, \mathbb{C}))$ :

$$
\begin{aligned}
H^{2,2}= & -\left(p^{0}\right)^{12}\left(p^{0}\right)^{21}+E_{1}(u)\left[-\left(p^{0}\right)^{12}\left(p^{1}\right)^{21}+\left(p^{0}\right)^{21}\left(p^{1}\right)^{12}\right]+ \\
& +\left(p^{1}\right)^{12}\left(p^{1}\right)^{21}\left[E_{1}^{\prime}(u)+E_{1}^{2}(u)\right]-2 v\left(p^{1}\right)^{11}, \\
H^{2,0}= & v^{2}+\left(\left(p^{1}\right)^{11}\right)^{2}\left[\frac{1}{6} E_{1}^{\prime \prime \prime}(u)+\left(E_{1}^{\prime}(u)\right)^{2}\right]+\left(p^{0}\right)^{12}\left(p^{0}\right)^{21} E_{1}^{\prime}(u)+ \\
& +\left[\left(p^{0}\right)^{12}\left(p^{1}\right)^{21}-\left(p^{0}\right)^{21}\left(p^{1}\right)^{12}\right]\left[E_{1}(u) E_{1}^{\prime}(u)+\frac{1}{2} E_{1}^{\prime \prime}(u)\right]- \\
& -\left(p^{1}\right)^{12}\left(p^{1}\right)^{21}\left[\left(E_{1}^{\prime}(u)\right)^{2}+E_{1}^{2}(u) E_{1}^{\prime}(u)+E_{1}(u) E_{1}^{\prime \prime}(u)+\frac{1}{3} E_{1}^{\prime \prime \prime}(u)\right],
\end{aligned}
$$

где $u, v \in \mathcal{H}$ - подалгебра Картана группы $S L(N, \mathbb{C}), E_{1}\left(u^{i j}\right) \equiv E_{1}\left(u^{i j}, \tau\right)$ - эллиптическая функция Эйзенштейна (приложение 1 ), определенная на комплексном торе $T^{2} \mathrm{c}$ модулем $\tau$ и связанная с $\zeta$-функцией Вейерштрасса: $E_{1}(z, \tau)=\zeta(z, \tau)+2 \zeta(1 / 2) z$.

Скобка Ли-Пуассона между переменными $u$ и $v$ задается формулой (11), а скобки меж ду переменными $p^{r}$ остаются такими же, как и в рациональном случае. Для эллиптического случая матрища Лакса при слиянии $r$ точек имеет вид: 
диагональная часть -

$$
L^{i i}=v^{i}+\left(p^{1}\right)^{i i} \tilde{k}_{2} E_{1}^{\prime}\left(z-x_{a}\right)+\cdots+\left(p^{r}\right)^{i i} \tilde{k}_{r} E_{1}^{(r)}\left(z-x_{a}\right),
$$

недиагональная часть -

$$
L^{i j}=\left(p^{0}\right)^{i j} \tilde{k}_{1} \varphi\left(u^{i j}, z-x_{a}\right)+\cdots+\left(p^{r}\right)^{i j} \tilde{k}_{r} \varphi^{(r)}\left(u^{i j}, z-x_{a}\right),
$$

где $\tilde{k}_{r}$ аналогичны $k_{r}$. Гамильтонианы в случае двух точек, выраженные через переменные, инвариантные относительно фиксации калибровки, даны в формуле (49), соответствующие коммутационные соотношения - формулы (52), (53), симметрии - формула $(70)$.

Симплектическая форма в случае слияния двух точек в рациональном и эллиптическом случаях описана в разделе 3 .

Результаты, полученные с применением предела Иноземцева. Гамильтонианы системы типа Тоды, полученные с применением предела Иноземцев (ПИ) из эллиптической системы Годена в случае слияния двух точек, а также соответствующие коммутационные соотношения указаны в разделе 5 (формулы $(82),(84)$ ). Отметим, что системы типа Тоды с нетривиальными коммутационными соотношениями, полученные с применением ПИ из эллиптической системы Годена, также изучались в работе [8].

Способы получения новых систем. Для получения интегрируемых систем из рациональной и эллиптической систем Годена используется процедура слияния точек. Основная идея этого способа состоит в нахож дении такого разложения переменных $p_{a}^{i j}$, при применении которого и после взятия предела $x_{b}-x_{a}=\varepsilon \rightarrow 0$, где $\varepsilon$ - параметр слияния (в данном случае коэффициент при $\varepsilon$ мы положили равным единице), возникали бы полюсы порядка выше первого у матрицы Лакса в точке $x_{a}$, что означает существование в новой системе такого же количества и гамильтонианов, и операторов Казимира, что и в начальной. Такая ситуация реализуется при переходе к новым переменным $p^{r} \mathrm{c}$ помошью градуированного разложения

$$
p_{a}=\alpha_{0} p^{0}+\alpha_{1} p^{1} \varepsilon^{-1}+\cdots+\alpha_{r} p^{r} \varepsilon^{-r},
$$

где $\alpha_{r}$ - некоторые параметры.

В настоящей работе используется также процедура, называемая ПИ [9]. Это способ получения интегрируемых систем типа Тоды (систем частиц с экспоненциальным типом взаимодействия) из эллиптических систем типа Годена (систем частиц с эллиптическим типом взаимодействия) путем комбинирования тригонометрического предела, бесконечного сдвига координат частиц и перенормировки констант связи. Технические подробности изложены в работах [9], [10]. 


\section{2. РАЦИОНАЛЬНАЯ И ЭЛЛИПТИЧЕСКАЯ СИСТЕМЫ ГОДЕНА}

Начнем с рациональной системы Годена. Рассмотрим $n$ отмеченных точек $x_{a}, a=$ $\overline{1, n}$ на $\mathbb{C} P^{1}$ и зададим в каждой точке орбиту коприсоединенного представления группы $S L(N, \mathbb{C})$. Координаты $p_{a}^{i j}$ являются функциями на соответствуюших орбитах. Скобка Ли-Пуассона от функций $p_{a}^{i j}$ (спинов) вьглядит следующим образом (приложение 2 ):

$$
\left\{p_{a}^{i j}, p_{b}^{k l}\right\}=\delta_{a b}\left(\delta^{i l} p_{a}^{k j}-\delta^{k j} p_{a}^{i l}\right) .
$$

Фазовое пространство - прямое произведение этих орбит, факторизованное по группе $S L(N, \mathbb{C})$, элементы которой не зависят от координаты на $\mathbb{C} P^{1}$. На этом фазовом пространстве определяется интегрируемая система, задаваемая гамильтонианами, коммутируюшими относительно скобки Ли-Пуассона (7) и имеюшими вид

$$
H_{a}=\sum_{b \neq a} \frac{\operatorname{Tr}\left(p_{a} p_{b}\right)}{x_{b}-x_{a}}
$$

Другие гамильтонианы этой системы также выражаются через следы произведений спинов и разностей координат отмеченных точек в некоторых степенях. Оператор Лакса задается формулой

$$
L(z)=\sum_{a} \frac{p_{a}}{z-x_{a}}
$$

где $z$ - координата на $\mathbb{C} P^{1}$.

Опишем эллиптический случай: вместо $\mathbb{C} P^{1}$ рассмотрим эллиптическую кривую. Интегрируемая система определена теперь на симплектическом факторпространстве $R$ [4], которое имеет вид

$$
R=(u, v) \times\left(\prod_{a} \mathcal{O}_{a} / / H\right)
$$

где $\mathcal{O}_{a}$ - орбита коприсоединенного представления группы $S L(N, \mathbb{C}), \quad H$ - подгруппа Картана, $u, v \in \mathcal{H}$ - подалгебра Картана.

$\mathrm{K}$ коммутационным соотношениям между $p_{a}^{i j}$ добавляются соотношения между $v, u$ и $p_{a}^{i j}$ :

$$
\left\{v^{i}, u^{j}\right\}=\delta^{i j}, \quad\left\{v^{k}, p_{a}^{i j}\right\}=0, \quad\left\{u^{k}, p_{a}^{i j}\right\}=0 .
$$

Наиболее известный из гамильтонианов эллиптической системы Годена описывает физическую систему из $n$ взаимодействуюших частиц:

$$
H=\sum_{i} \frac{1}{2}\left(v^{i}\right)^{2}+\sum_{i \neq j} \operatorname{Tr} p^{2} E_{2}\left(u^{i j}\right),
$$

где $v_{i}$ - импульсы, $u^{i j}=u^{i}-u^{j}$ - разность координат частиц, $E_{2}\left(u^{i j}\right) \equiv E_{2}\left(u^{i j}, \tau\right)-$ потенциал взаимодействия частиц, представленный эллиптической функцией Эйзенш-

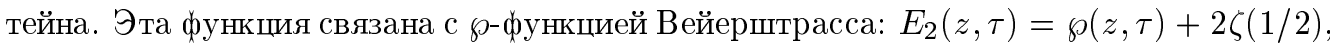


где $z$ - координата на торе. Функция $E_{2}(z, \tau)$ выражается через $\theta$-функцию (приложение 1) следуюшим образом: $E_{2}(z, \tau)=-\partial_{z}^{2} \ln (\theta(z, \tau))$.

Матриша Лакса в эллиптическом случае выглядит следуюшим образом:

диагональная часть -

$$
L^{i i}=v^{i}+\sum_{a} p_{a}^{i i} E_{1}\left(z-x_{a}\right)
$$

недиагональная часть -

$$
L^{i j}=\sum_{a} p_{a}^{i j} \exp \left[-2 \pi i u^{i j} \frac{\left(z-x_{a}\right)-\left(\bar{z}-\bar{x}_{a}\right)}{\tau-\bar{\tau}}\right] \varphi\left(u^{i j}, z-x_{a}\right)
$$

где

$$
\begin{gathered}
\varphi\left(u^{i j}, z-x_{a}\right)=\frac{\theta\left(u^{i j}+z-x_{a}\right) \theta^{\prime}(0)}{\theta\left(u^{i j}\right) \theta\left(z-x_{a}\right)} \\
\text { 3. СИСТЕМЫ, ОБРАЗУЮЩИЕСЯ В РЕЗУЛЬТАТЕ } \\
\text { ПРЕОБРАЗОВАНИЯ РАЦИОНАЛЬНОЙ СИСТЕМЫ ГОДЕНА }
\end{gathered}
$$

\section{3. СИСТЕМЫ, ОБРАЗУЮЩИЕСЯ В РЕЗУЛЬТАТЕ ПРЕОБРАЗОВАНИЯ РАЦИОНАЛЬНОЙ СИСТЕМЫ ГОДЕНА}

Рассмотрим случай слияния двух точек из обшего количества $n>4$, проведем следующее преобразование координат $x$ и разложение переменных $p$ :

$$
x_{b}=x_{a}+\varepsilon, \quad p_{a}=\alpha_{0} p^{0}+\alpha_{1} p^{1} \varepsilon^{-1}, \quad p_{b}=\beta_{0} p^{0}+\beta_{1} p^{1} \varepsilon^{-1},
$$

где $\alpha_{r}, \beta_{r}$ - некоторые параметры. Подставим (16) в матрицу оператора Лакса (9) и рассмотрим предел при $\varepsilon \rightarrow 0$ :

$$
L=-\frac{\alpha_{0} p^{0}+\alpha_{1} p^{1} \varepsilon^{-1}}{z-x_{a}}+\frac{\beta_{0} p^{0}+\beta_{1} p^{1} \varepsilon^{-1}}{z-x_{a}-\varepsilon}+\sum_{c \neq a, b} \frac{p_{c}}{z-x_{c}} .
$$

При вычислении мы должны вводить дополнительные условия для параметров. Причина их возникновения - требование отсутствия сингулярностей от степеней $\varepsilon^{-1}$ при $\varepsilon \rightarrow 0$. В данном случае имеем одно условие $\alpha_{1}+\beta_{1}=0$; положив $\alpha_{0}=1, \alpha_{1}=-1$, $\beta_{0}=0, \beta_{1}=1$, получим

$$
L_{\text {new }}=\frac{p^{1}}{\left(z-x_{a}\right)^{2}}+\frac{p^{0}}{z-x_{a}}+\sum_{c \neq a, b} \frac{p_{c}}{z-x_{c}}
$$

Разложение $\left\langle L_{\text {new }}^{2}\right\rangle$ по $\left(z-x_{a}\right)^{-m}$ имеет вид

$$
\begin{aligned}
\left\langle L_{\text {new }}^{2}\right\rangle= & H_{1}^{2,4}\left(z-x_{a}\right)^{-4}+H_{1}^{2,3}\left(z-x_{a}\right)^{-3}+H_{1}^{2,2}\left(z-x_{a}\right)^{-2}+H_{1}^{2,1}\left(z-x_{a}\right)^{-1}+ \\
& +\sum_{c \neq a, b}\left(H_{c}^{2,2}\left(z-x_{c}\right)^{-2}+H_{c}^{2,1}\left(z-x_{c}\right)^{-1}\right)
\end{aligned}
$$


где

$$
\begin{aligned}
H_{1}^{2,4} & =\left\langle\left(p^{1}\right)^{2}\right\rangle, \quad H_{1}^{2,3}=2\left\langle p^{1} p^{0}\right\rangle, \\
H_{1}^{2,2} & =\left\langle\left(p^{0}\right)^{2}\right\rangle+2 \sum_{c \neq a, b} \frac{\left\langle p^{1} p_{c}\right\rangle}{x_{a}-x_{c}}, \\
H_{1}^{2,1} & =\sum_{c \neq a, b}\left[\frac{\left\langle p^{0} p_{c}\right\rangle}{x_{a}-x_{c}}-\frac{\left\langle p^{1} p_{c}\right\rangle}{\left(x_{a}-x_{c}\right)^{2}}\right], \quad H_{c}^{2,2}=\left\langle\left(p_{c}\right)^{2}\right\rangle, \\
H_{c}^{2,1} & =2 \frac{\left\langle p^{1} p_{c}\right\rangle}{\left(x_{c}-x_{a}\right)^{2}}+\frac{\left\langle p^{0} p_{c}\right\rangle}{x_{c}-x_{a}}+\sum_{d \neq c, a, b} \frac{\left\langle p_{c} p_{d}\right\rangle}{x_{c}-x_{d}} .
\end{aligned}
$$

Здесь $H_{1}^{2,4}, H_{1}^{2,3}, H_{c}^{2,2}$ - операторы Казимира.

Условие $\sum_{a} p_{a}=0$, являюшееся уравнением отображения момента по действию группы остаточных преобразований, изменится, так как после преобразований $p_{a}$ и $p_{b}$ получим

$$
p^{0}+\sum_{c \neq a, b} p_{c}=0 .
$$

У новой системы количество гамильтонианов и операторов Казимира не изменилось: вместо двух гамильтонианов и двух операторов Казимира рациональной системы Годена, относяшихся к точкам $x_{a}, x_{b}$, появились операторы Казимира $H_{1}^{2,4}, H_{1}^{2,3}$ и гамильтонианы $H_{1}^{2,2}, H_{1}^{2,1}$. Можно вычислить коммутационные соотношения для новых переменных; в результате получим

$$
\begin{aligned}
& \left\{\left(p^{1}\right)^{i j},\left(p^{1}\right)^{k l}\right\}=0, \\
& \left\{\left(p^{0}\right)^{i j},\left(p^{0}\right)^{k l}\right\}=\delta^{i l}\left(p^{0}\right)^{k j}-\delta^{k j}\left(p^{0}\right)^{i l}, \\
& \left\{\left(p^{0}\right)^{i j},\left(p^{1}\right)^{k l}\right\}=\delta^{i l}\left(p^{1}\right)^{k j}-\delta^{k j}\left(p^{1}\right)^{i l} .
\end{aligned}
$$

Схематично это может быть представлено как

$$
\left(p^{1}, p^{1}\right) \rightarrow 0, \quad\left(p^{0}, p^{0}\right) \rightarrow p^{0}, \quad\left(p^{0}, p^{1}\right) \rightarrow p^{1} .
$$

Используя элементы $p^{0}, p^{1}$, можно составить матрицы вида

$$
P=\left(\begin{array}{cc}
p^{0} & p^{1} \\
0 & p^{0}
\end{array}\right)
$$

которые образуют параболическую алгебру. Можно показать, что коммутационные соотношения между $p^{r}$ с учетом позиций $p^{r}$ в $P$ (в случае $p^{r} \in s l(2, \mathbb{C})$ ), т.е. при рассмотрении $p^{r}$ как элементов $P$, выглядят следуюшим образом (приложение 2 ):

$$
\begin{aligned}
& \left\{\left(p^{0}\right)^{i j},\left(p^{1}\right)^{k l}\right\}=\delta^{i l}\left(p^{1}\right)^{k, j+N}-\delta^{k j}\left(p^{1}\right)^{i, l+N}, \\
& \left\{\left(p^{0}\right)^{i j},\left(p^{0}\right)^{k l}\right\}=\frac{1}{2}\left[\delta^{i l}\left(\left(p^{0}\right)^{k j}+\left(p^{0}\right)^{k+N, j+N}\right)-\delta^{k j}\left(\left(p^{0}\right)^{i l}+\left(p^{0}\right)^{i+N, l+N}\right)\right],
\end{aligned}
$$

где $N=2$. Петлевое разложение матрицы $P$ в случае двух точек будет давать многочлены вида $p^{0}+p^{1} \varepsilon^{-1}$. Изоморфизм можно установить, сравнивая коммутационные соотношения для элементов матриц и многочленов. 
Обобшим вышесказанное на случай большего количества точек. Рассматривая преобразования (16) для $x$ и $p$ в случае слияния первых (для определенности) $r$ точек из рассматриваемых $n$, получаем выражение для $L_{\text {new }}$, не зависящее от последовательности слияния точек, но с точностью до коэффициентов зависящее от их количества:

$$
L_{\mathrm{new}}=\frac{p^{r} k_{r}}{\left(z-x_{a}\right)^{r}}+\cdots+\frac{p^{1} k_{1}}{\left(z-x_{a}\right)^{2}}+\frac{p^{0} k_{0}}{z-x_{a}}+\sum_{c \neq a, b} \frac{p_{c}}{z-x_{c}}
$$

Соответствуюшие матрищы $P$, образуюшие параболическую алгебру, имеют вид

$$
P=\left(\begin{array}{ccccc}
p^{0} & p^{1} & \ldots & p^{n-1} & p^{n} \\
0 & p^{0} & \ldots & p^{n-2} & p^{n-1} \\
\vdots & \vdots & \ddots & \vdots & \vdots \\
0 & 0 & \ldots & p^{0} & p^{1} \\
0 & 0 & \ldots & 0 & p^{0}
\end{array}\right)
$$

Коммутационные соотношения схематически (с точностью до коэффициентов) можно представить в виде таблицы 1.

ТАБЛИЦА 1

\begin{tabular}{|c|c|c|c|c|c|c|}
\hline$p$ & $p^{0}$ & $p^{1}$ & $p^{2}$ & $\cdots$ & $p^{n-1}$ & $p^{n}$ \\
\hline$p^{0}$ & $p^{0}$ & $p^{1}$ & $p^{2}$ & $\cdots$ & $p^{n-1}$ & $p^{n}$ \\
\hline$p^{1}$ & $p^{1}$ & $p^{2}$ & $p^{3}$ & $\cdots$ & $p^{n}$ & 0 \\
\hline$p^{2}$ & $p^{2}$ & $p^{3}$ & $p^{4}$ & $\cdots$ & 0 & 0 \\
\hline$\vdots$ & $\vdots$ & $\vdots$ & $\vdots$ & $\ddots$ & $\vdots$ & $\vdots$ \\
\hline$p^{n-1}$ & $p^{n-1}$ & $p^{n}$ & 0 & $\cdots$ & 0 & 0 \\
\hline$p^{n}$ & $p^{n}$ & 0 & 0 & $\cdots$ & 0 & 0 \\
\hline
\end{tabular}

Здесь пересечение столбца и строки - результат коммутационных соотношений.

Симплектическая форма в случае слияния точек. Рассмотрим симплектическую форму системы Хитчина в соответствии с работами [2]-[5] в случае $n$ отмеченных точек на $\Sigma=\mathbb{C} P^{1}$, выделив две для последующего слияния:

$$
\begin{aligned}
\Omega= & \int_{\Sigma}\langle\delta A \wedge \delta \bar{A}\rangle+\sum_{a}\left\langle p_{a}, g_{a}^{-1} \delta g_{a}\right\rangle= \\
= & \int_{\Sigma}\left[\langle\delta A \wedge \delta \bar{A}\rangle+\delta\left(z-x_{a}\right)\left\langle p_{a}, g_{a}^{-1} \delta g_{a}\right\rangle+\delta\left(z-x_{b}\right)\left\langle p_{b}, g_{b}^{-1} \delta g_{b}\right\rangle+\right. \\
& \left.+\sum_{c \neq a, b} \delta\left(z-x_{c}\right)\left\langle p_{c}, g_{c}^{-1} \delta g_{c}\right\rangle\right],
\end{aligned}
$$


где $\langle\ldots\rangle$ означает форму Киллинга, $\delta$ - оператор внешнего дифффееренцирования, $A$ и $\bar{A}$ означают соответственно голоморфную и антиголоморфную части связности, $\left(p_{a}, g_{a}\right) \in$ $T^{*} G_{a}$ (кокасательное расслоение) в отмеченных точках: $p_{a} \in \operatorname{Lie}^{*}\left(G_{a}\right), g_{a} \in G_{a}$. Симплектическая форма инвариантна относительно действия группы:

$$
G_{1}=\left\{f(z, \bar{z}) \in C^{\infty}(\Sigma), G\right\}
$$

Соответствуюшие калибровочные преобразования имеют вид

$$
\begin{aligned}
A & =f L f^{-1}+f \partial f^{-1}, \\
\bar{A} & =f \bar{L} f^{-1}+f \bar{\partial} f^{-1}, \\
p_{a} & =f_{a} p_{a} f_{a}^{-1}, \quad g_{a}=g_{a} f_{a}^{-1} .
\end{aligned}
$$

Уравнение отображения момента будет иметь вид

$$
\bar{\partial} L=\delta\left(z-x_{a}\right) p_{a}+\delta\left(z-x_{b}\right) p_{b}+\sum_{c \neq a, b} \delta\left(z-x_{t}\right) p_{t} .
$$

Выполняя преобразования (16), получим

$$
\bar{\partial} L=\delta\left(z-x_{a}\right) p^{0}+\delta^{\prime}\left(z-x_{a}\right) p^{1}+\sum_{c \neq a, b} \delta\left(z-x_{c}\right) p_{c} .
$$

Решая это уравнение, получим оператор Лакса с полюсом второго порядка. Теперь найдем такое преобразование формы $\Omega$, которое позволит получить новую форму, даюшую уравнение отображения момента (32). Введем логарифмические координаты $\ln g$ на группе $S L(N, \mathbb{C})$, являюшиеся координатами на алгебре $s l(N, \mathbb{C})$; тогда $\ln g_{a} \equiv X_{a}$, $\ln g_{b} \equiv X_{b}$ и $g_{a}^{-1} \delta g_{a} \equiv \delta X_{a}, g_{b}^{-1} \delta g_{b} \equiv \delta X_{b}$. Рассмотрим следуюшие преобразования:

$$
X_{a}=X^{0} \varepsilon^{-1}-X^{1}, \quad X_{b}=-X^{0} \varepsilon^{-1}-X^{1} .
$$

Определим спаривание между коалгеброй (элементы $P$ ) и алгеброй (элементы $X$ ) как $\operatorname{Res}_{-1}\langle P X\rangle$. Подставим преобразования (16), положив $\alpha_{0}=1, \alpha_{1}=-1, \beta_{0}=0, \beta_{1}=1$, и (33) в выражение для $\Omega$. Разлагая по $\varepsilon$, приводя подобные и возврашаясь к обозначениям $\ln g_{0} \equiv X^{0}$, получим

$\Omega=\int_{\Sigma}\left[\langle\delta A \wedge \delta \bar{A}\rangle+\delta\left(z-x_{a}\right)\left\langle p^{0}, g_{0}^{-1} \delta g_{0}\right\rangle+\delta^{\prime}\left(z-x_{a}\right)\left\langle p^{1}, g_{0}^{-1} \delta g_{0}\right\rangle\right]+\sum_{c \neq a, b}\left\langle p_{c}, g_{c}^{-1} \delta g_{c}\right\rangle$.

Отсюда непосредственно получим (32). 


\section{4. СИСТЕМЫ, ОБРАЗУЮШИЕСЯ В РЕЗУЛЬТАТЕ ПРЕОБРАЗОВАНИЯ ЭЛЛИПТИЧЕСКОЙ СИСТЕМЫ ГОДЕНА}

Гамильтонианы системы Годена в случае двух точек. Начнем, как и в рациональном случае, со слияния двух точек. Рассмотрим две отмеченные точки $x_{a}$ и $x_{b}$. Как уже говорилось во введении, интегрируемая система определена на симплектическом факторпространстве $R[4]$, которое в случае двух точек имеет вид

$$
R=(u, v) \times\left(\mathcal{O}_{a} \times \mathcal{O}_{b} / / H\right)
$$

где $\mathcal{O}_{a}$ и $\mathcal{O}_{b}$ - орбиты коприсоединенного представления группы $S L(N, \mathbb{C}), H$ - подгруппа Картана.

Уравнение отображения момента, соответствуюшее действию группы остаточных преобразований, имеет вид

$$
\sum_{a} p_{a}^{i i}=0
$$

Рассмотрим случай $N=2$, т.е. $p \in \operatorname{sl}(2, \mathbb{C})$. Получим гамильтонианы, разлагая $\left\langle L^{2}\right\rangle$ по базису функций Эйзенштейна, где $L$ - матрица Лакса в голоморфном представлении:

диагональная часть -

$$
L^{i i}=v^{i}+\sum_{a} p_{a}^{i i} E_{1}\left(z-x_{a}\right)
$$

недиагональная часть -

$$
L^{i j}=\sum_{a} p_{a}^{i j} \varphi\left(u^{i j}, z-x_{a}\right),
$$

где

$$
\varphi\left(u^{i j}, z-x_{a}\right)=\frac{\theta\left(u^{i j}+z-x_{a}\right) \theta^{\prime}(0)}{\theta\left(u^{i j}\right) \theta\left(z-x_{a}\right)} .
$$

В случае двух точек получим

$$
\begin{aligned}
\left\langle L^{2}\right\rangle= & \left(v^{1}+p_{a}^{11} E_{1}\left(z-x_{a}\right)+p_{b}^{11} E_{1}\left(z-x_{b}\right)\right)^{2}+ \\
& +\left(v^{2}+p_{a}^{22} E_{1}\left(z-x_{a}\right)+p_{b}^{22} E_{1}\left(z-x_{b}\right)\right)^{2}+ \\
& +p_{a}^{12} p_{a}^{21}\left(-E_{1}^{\prime}\left(z-x_{a}\right)+E_{1}^{\prime}\left(u^{12}\right)\right)+p_{b}^{12} p_{b}^{21}\left(-E_{1}^{\prime}\left(z-x_{b}\right)+E_{1}^{\prime}\left(u^{12}\right)\right)+ \\
& +2 p_{a}^{12} p_{b}^{21} \varphi\left(u^{12}, z-x_{a}\right) \varphi\left(u^{21}, z-x_{b}\right)+ \\
& +2 p_{a}^{21} p_{b}^{12} \varphi\left(u^{21}, z-x_{a}\right) \varphi\left(u^{12}, z-x_{b}\right) .
\end{aligned}
$$

Анализируя нули и полюсы в $\left\langle L^{2}\right\rangle$ (см. приложение 3 ), получим разложение

$$
\left\langle L^{2}\right\rangle=\sum_{a}\left(E_{1}^{\prime}\left(z-x_{a}\right) H_{a}^{2,2}+E_{1}\left(z-x_{a}\right) H_{a}^{2,1}\right)+H_{a}^{2,0}
$$


где

$$
\begin{aligned}
H_{a}^{2,2}= & \left\langle p_{a}^{2}\right\rangle, \\
H_{a}^{2,1}= & v^{1} p_{a}^{11}+v^{2} p_{a}^{22}+\left[p_{a}^{11} p_{b}^{11}+p_{a}^{22} p_{b}^{22}\right] E_{1}\left(x_{a}-x_{b}\right)+ \\
& +2 p_{a}^{12} p_{b}^{21} \frac{\theta\left(u^{12}+x_{b}-x_{a}\right) \theta^{\prime}(0)}{\theta\left(u^{12}\right) \theta\left(x_{a}-x_{b}\right)}+2 p_{a}^{21} p_{b}^{12} \frac{\theta\left(u^{12}+x_{a}-x_{b}\right) \theta^{\prime}(0)}{\theta\left(u^{12}\right) \theta\left(x_{b}-x_{a}\right)}, \\
H^{2,0}= & \left(v^{1}\right)^{2}+\left(v^{2}\right)^{2}-\left[p_{a}^{11} p_{b}^{11}+p_{a}^{22} p_{b}^{22}\right]\left(E_{1}^{\prime}\left(x_{a}-x_{b}\right)+E_{1}^{2}\left(x_{a}-x_{b}\right)\right)+ \\
& +\left[p_{a}^{12} p_{a}^{21}+p_{b}^{12} p_{b}^{21}\right] E_{1}^{\prime}\left(u^{12}\right)+ \\
& +2 p_{a}^{12} p_{b}^{21}\left(E_{1}\left(u^{12}\right)-E_{1}\left(u^{12}+x_{b}-x_{a}\right)\right) \frac{\theta\left(u^{12}+x_{b}-x_{a}\right) \theta^{\prime}(0)}{\theta\left(u^{12}\right) \theta\left(x_{a}-x_{b}\right)}+ \\
& +2 p_{a}^{21} p_{b}^{12}\left(E_{1}\left(u^{12}\right)-E_{1}\left(u^{12}+x_{a}-x_{b}\right)\right) \frac{\theta\left(u^{12}+x_{a}-x_{b}\right) \theta^{\prime}(0)}{\theta\left(u^{12}\right) \theta\left(x_{b}-x_{a}\right)} .
\end{aligned}
$$

Вычислим размерность фазового пространства интегрируемой системы. Всего переменных восемь: $u^{12}, v^{1}, p_{a}^{11}, p_{a}^{12}, p_{a}^{21}, p_{b}^{11}, p_{b}^{12}, p_{b}^{21}$, что согласовано с двумя операторами Казимира и тремя гамильтонианами. Выберем орбиты, т.е. фиксируем значения операторов Казимира $H_{a}^{2,2}=\left(\lambda_{a}\right)^{2}$ и $H_{b}^{2,2}=\left(\lambda_{b}\right)^{2}$, и, учитывая уравнение отображения момента (36), получим

$$
\left(p_{a}^{11}\right)^{2}+p_{a}^{12} p_{a}^{21}=\left(\lambda_{a}\right)^{2}, \quad\left(p_{b}^{11}\right)^{2}+p_{b}^{12} p_{b}^{21}=\left(\lambda_{b}\right)^{2}, \quad p_{a}^{11}+p_{b}^{11}=0 .
$$

Заметим, что группа остаточных преобразований в обшем случае (не голоморфном) состоит из двоякопериодических функций от $(z, \bar{z})$ на диагонали, так как они не меняют фиксацию калибровки в уравнении отображения момента (см. симметрии эллиптической системы Годена). Разлагая эти функции в ряд Фурье, мы получим базис в пространстве остаточных преобразований.

Выберем теперь калибровку по действию группы остаточных преобразований - диагональных матриц, не зависящих от $(z, \bar{z}),-$ подгруппы Картана, соответствуюшей отображению момента (36). Из сказанного выше следует, что, фиксируя калибровку, мы рассматриваем такие функции, которые имеют только нулевую гармонику, т.е. первые члены $c_{0}=e^{+\alpha}$ в разложении - константы, образуюшие однопараметрическое семейство. Введем переменные, инвариантные относительно фиксации калибровки:

$$
p_{a}^{12} p_{a}^{21} \equiv x, \quad p_{b}^{12} p_{b}^{21} \equiv y, \quad p_{a}^{12} p_{b}^{21} \equiv z_{1}, \quad p_{b}^{12} p_{a}^{21} \equiv z_{2}, \quad p_{a}^{11}, \quad p_{b}^{22}, \quad u, \quad v .
$$

К уравнениям (45) добавится уравнение связи между переменными; получим

$$
\left(p_{a}^{11}\right)^{2}+x=\left(\lambda_{a}\right)^{2}, \quad\left(p_{b}^{11}\right)^{2}+y=\left(\lambda_{b}\right)^{2}, \quad p_{a}^{11}+p_{b}^{11}=0, \quad x y=z_{1} z_{2} .
$$

Отсюда видно, что мы можем шесть переменных (все, кроме $u$ и $v$ ) выразить через две, например, $z_{1}$ и $z_{2}$. Таким образом мы получим четырехмерное фазовое пространство, что соответствует двум гамильтонианам. Заметим, что гамильтонианы как функции на этом фазовом пространстве будут инвариантными относительно действия подгруппы остаточных преобразований, так как фазовое пространство - факторпространство по действию этой подгруппы. 
Гамильтонианы системы, полученной слиянием двух точек. Реализуем слияние точек в соответствии с преобразованиями (16). Заметим, что коммутационные соотношения между новыми переменными $\left(p^{0}\right)^{i j}$ и $\left(p^{1}\right)^{i j}$ не зависят от эллиптических функций и остаются такими же, как и в случае рациональной системы Годена. Основное, что добавляется при вычислении пределов - разложение эллиптических функций в ряды по $\varepsilon$. В случае $p \in \operatorname{sl}(2, \mathbb{C})$ после вычислений, положив $u^{12} \equiv u$ и $v^{1}=-v^{2}=v$, получим (приложение 4)

$$
\frac{1}{2}\left\langle L_{\text {new }}^{2}\right\rangle=E_{1}^{\prime \prime \prime}\left(z-x_{a}\right) H^{2,4}+E_{1}^{\prime \prime}\left(z-x_{a}\right) H^{2,3}+E_{1}^{\prime}\left(z-x_{a}\right) H^{2,2}+H^{2,0}
$$

где

$$
\begin{aligned}
H^{2,4}= & -\frac{1}{6} \frac{b c}{a}-\frac{1}{6} g^{2}=-\frac{1}{12}\left\langle\left(p^{1}\right)^{2}\right\rangle, \quad H^{2,3}=\frac{1}{2}(b+c)=\frac{1}{2}\left\langle p^{0} p^{1}\right\rangle \\
H^{2,2}= & -a+(c-b) E_{1}(u)+\frac{b c}{a}\left[E_{1}^{\prime}(u)+E_{1}(u)^{2}\right]-2 v g \\
H^{2,0}= & v^{2}+g^{2}\left[\frac{1}{6} E_{1}^{\prime \prime \prime}(u)+\left(E_{1}^{\prime}(u)\right)^{2}\right]+a E_{1}^{\prime}(u)+(b-c)\left[\frac{1}{2} E_{1}^{\prime \prime}(u)+E_{1}^{\prime}(u) E_{1}(u)\right]- \\
& -\frac{b c}{a}\left[\frac{1}{3} E_{1}^{\prime \prime \prime}(u)+\left(E_{1}^{\prime}(u)\right)^{2}+E_{1}^{\prime \prime}(u) E_{1}(u)+E_{1}^{\prime}(u) E_{1}(u)^{2}\right] .
\end{aligned}
$$

Здесь мы ввели дополнительно к $v$ и $u$ переменные, инвариантные относительно фиксации калибровки:

$$
\begin{aligned}
& \left(p^{0}\right)^{12}\left(p^{0}\right)^{21}=a, \quad\left(p^{0}\right)^{12}\left(p^{1}\right)^{21}=b, \\
& \left(p^{1}\right)^{12}\left(p^{0}\right)^{21}=c, \quad\left(p^{1}\right)^{12}\left(p^{1}\right)^{21}=d=\frac{b c}{a}, \quad\left(p^{1}\right)^{11}=g .
\end{aligned}
$$

Вычислим размерность фазового пространства полученной системы. Всего переменных в $(u, v) \times\left(p^{r}\right)^{i j}$-пространстве восемь; $H^{2,4}$ и $H^{2,3}$ - операторы Казимира. Отображение момента по действию группы остаточных преобразований теперь имеет вид $\left(p^{0}\right)^{11}=0$. Фиксация операторов Казимира дает уравнения

$$
\frac{b c}{a}+g^{2}=\lambda_{0}, \quad b+c=\lambda_{1}
$$

Таким образом мы получим четырехмерное фазовое пространство и два гамильтониана. Скобки Пуассона в новых переменных принимают вид

$$
\begin{array}{lll}
\{v, u\}=1, & \{c, b\}=0, \quad\{a, g\}=c-b, \\
\{c, a\}=-2 a g, \quad\{b, a\}=2 a g, & \{b, g\}=-\frac{b c}{a}, \quad\{c, g\}=\frac{b c}{a} .
\end{array}
$$

Приведем также скобки Пуассона для $d \equiv b c / a$ :

$$
\left\{\frac{b c}{a}, a\right\}=2 g(b-c), \quad\left\{\frac{b c}{a}, g\right\}=0, \quad\left\{\frac{b c}{a}, b\right\}=-2 g \frac{b c}{a}, \quad\left\{\frac{b c}{a}, c\right\}=2 g \frac{b c}{a} .
$$


Симметрии эллиптической системы Годена. Гамильтонианы, определенные на фазовом пространстве, должны быть инвариантны относительно действия группы остаточных преобразований - группы Бернштейна-Шварцмана - полупрямого произведения группы Вейля и сдвигов решетки.

Будем рассматривать (в соответствии с работой [4]) калибровочные преобразования, не меняюшие фиксашию калибровки $\bar{L}=\operatorname{diag}(s l(2, \mathbb{C}))$, выбранной в уравнении отображения момента:

$$
\bar{\partial} L+\frac{2 \pi i}{\tau-\bar{\tau}}[\bar{L}, L]=2 \pi i\left(\delta^{2}\left(x_{a}\right) p_{a}+\delta^{2}\left(x_{b}\right) p_{b}\right)
$$

с граничными условиями

$$
L(z+1)=L(z), \quad L(z+\tau)=L(z),
$$

где

$$
\bar{L}=\left(\begin{array}{cc}
u^{1} & 0 \\
0 & u^{2}
\end{array}\right) .
$$

Тогда группа остаточных преобразований в общем случае состоит из двоякопериодических функций от $(z, \bar{z})$ со значениями в $\operatorname{diag}(s l(2, \mathbb{C}))$, так как они не меняют фиксацию калибровки в уравнении отображения момента. Разлагая эти функции в ряд Фурье, мы получим базис в пространстве остаточных преобразований - набор гармоник вида

$$
f^{i}=\exp \left[2 \pi i\left(m^{i} \frac{z-\bar{z}}{\tau-\bar{\tau}}+n^{i} \frac{\tau \bar{z}-\bar{\tau} z}{\tau-\bar{\tau}}\right)\right], \quad m^{i}, n^{i} \in \mathbb{Z} .
$$

Далее рассмотрим преобразование $\bar{L}$ под действием базисных функций:

$$
2 \pi i \frac{1}{\tau-\bar{\tau}}\left(\begin{array}{cc}
u^{1} & 0 \\
0 & u^{2}
\end{array}\right) \rightarrow f 2 \pi i \frac{1}{\tau-\bar{\tau}}\left(\begin{array}{cc}
u^{1} & 0 \\
0 & u^{2}
\end{array}\right) f^{-1}+f \bar{\partial} f^{-1},
$$

где

$$
f=\left(\begin{array}{cc}
f^{1} & 0 \\
0 & f^{2}
\end{array}\right) .
$$

Отсюда мы получим закон преобразования для $u^{i}$ :

$$
u^{i} \rightarrow u^{i}+m^{i}-n^{i} \tau .
$$

Рассмотрим преобразование $u^{12}$ в виде $u^{12} \rightarrow u^{12}+1=u^{1}-u^{2}+1$. Можно представить это преобразование как два: $u^{1} \rightarrow u^{1}+1$ и $u^{2} \rightarrow u^{2}$, тогда $f$ примет вид $\left(m^{1}=1, n^{1}=0\right)$

$$
f=\left(\begin{array}{cc}
f^{1} & 0 \\
0 & 1
\end{array}\right), \quad f^{1}=\exp \left[2 \pi i \frac{z-\bar{z}}{\tau-\bar{\tau}}\right] .
$$

Соответственно можно определить действие $f$ на $p_{a}$ :

$$
p_{a} \rightarrow f_{a} p_{a} f_{a}^{-1}=\left(\begin{array}{cc}
p_{a}^{11} & p_{a}^{12} f_{a}^{1} \\
p_{a}^{21}\left(f_{a}^{1}\right)^{-1} & p_{a}^{22}
\end{array}\right) .
$$


Мы получили закон преобразования для $p_{a}^{i j}$ :

$$
p_{a}^{i i} \rightarrow p_{a}^{i i}, \quad p_{a}^{12} \rightarrow p_{a}^{12} \exp \left[2 \pi i \frac{x_{a}-\bar{x}_{a}}{\tau-\bar{\tau}}\right], \quad p_{a}^{21} \rightarrow p_{a}^{21} \exp \left[-2 \pi i \frac{x_{a}-\bar{x}_{a}}{\tau-\bar{\tau}}\right] .
$$

Рассуждая аналогично, можно получить похожую формулу для $u^{1} \rightarrow u^{1}+\tau$, и в общем случае

$$
\begin{gathered}
u^{i j} \rightarrow u^{i j}+m^{i}-n^{i} \tau, \\
p_{a}^{i i} \rightarrow p_{a}^{i i}, \\
p_{a}^{i j} \rightarrow p_{a}^{i j} \exp \left[2 \pi i m^{i} \frac{x_{a}-\bar{x}_{a}}{\tau-\bar{\tau}}+2 \pi i n^{i} \frac{\bar{\tau} x_{a}-\bar{x}_{a} \tau}{\tau-\bar{\tau}}\right], \\
\varphi\left(u^{i j}, z-x_{a}\right) \rightarrow \varphi\left(u^{i j}, z-x_{a}\right) \exp \left[+2 \pi i n^{i}\left(z-x_{a}\right)\right] .
\end{gathered}
$$

Симметрии системы, полученной слиянием точек. Сделаем калибровочные преобразования переменных $L, \bar{L}, p_{a}^{i j}$ в уравнении отображения момента, используя

$$
\tilde{f}=\left(\begin{array}{cc}
\exp \left[-2 \pi i u^{1} \frac{z-\bar{z}}{\tau-\bar{\tau}}\right] & 0 \\
0 & \exp \left[-2 \pi i u^{2} \frac{z-\bar{z}}{\tau-\bar{\tau}}\right]
\end{array}\right) .
$$

Тогда мы получим уравнение отображения момента в виде

$$
\bar{\partial} L=2 \pi i\left(\delta^{2}\left(x_{a}\right) p_{a}+\delta^{2}\left(x_{b}\right) p_{b}\right)
$$

с граничными условиями для матрицы оператора Лакса:

$$
L^{i j}(z+1)=L^{i j}(z), \quad L^{i j}(z+\tau)=L^{i j}(z) \exp \left(-2 \pi i u^{i j}\right) .
$$

Поэтому недиагональная часть матрицы оператора Лакса примет голоморфный вид

$$
L^{i j}=p_{a}^{i j} \varphi\left(u^{i j}, z-x_{a}\right)+p_{b}^{i j} \varphi\left(u^{i j}, z-x_{b}\right) .
$$

Чтобы найти преобразования новых переменных $p_{a}^{i j}$ при $u^{i j} \rightarrow u^{i j}+m^{i}-n^{i} \tau$, нужно выразить их через старые переменные: $\left(p_{a}\right)_{\text {new }}^{i j}=p_{a}^{i j} \tilde{f}^{i j}\left(\tilde{f}^{j i}\right)^{-1}$, и получить суммарное преобразование. Проделав это, получим преобразования для переменных в голоморфной калибровке:

$$
\begin{aligned}
& u^{i j} \rightarrow u^{i j}+m^{i}-n^{i} \tau, \\
& p_{a}^{i j} \rightarrow p_{a}^{i j} \exp \left(-2 \pi i n^{i} x_{a}\right) .
\end{aligned}
$$

Теперь мы можем рассмотреть преобразования системы, полученной слиянием точек. Для этого в матрице оператора Лакса в голоморфной калибровке сделаем преобразования перешкалирования $p_{a}^{i j}$ и одновременно преобразования переменных при сдвижке $u^{i j}$. Получим измененное действие группы Бернштейна-Шварцмана на новые переменные $\left(p^{r}\right)^{i j}, \varphi\left(u^{i j}, z-x_{a}\right)$ и производные $\varphi\left(u^{i j}, z-x_{a}\right)$ по $\left(z-x_{a}\right)$ :

$$
\begin{gathered}
u^{i j} \equiv u \rightarrow u+m-n \tau, \\
\left(p^{0}\right)^{i j} \rightarrow\left(p^{0}\right)^{i j}-2 \pi i n\left(p^{1}\right)^{i j}, \quad\left(p^{1}\right)^{i j} \rightarrow\left(p^{1}\right)^{i j}, \quad\left(p^{1}\right)^{i i} \rightarrow\left(p^{1}\right)^{i i}, \\
\varphi\left(u, z-x_{a}\right) \rightarrow \varphi\left(u, z-x_{a}\right) \exp \left[+2 \pi i n\left(z-x_{a}\right)\right], \\
\varphi^{\prime}\left(u, z-x_{a}\right) \rightarrow\left[-2 \pi i n \varphi\left(u, z-x_{a}\right)+\varphi^{\prime}\left(u, z-x_{a}\right)\right] \exp \left[+2 \pi i n\left(z-x_{a}\right)\right] .
\end{gathered}
$$


Матрица оператора Лакса новой системы. Рассмотрим выражения для матрицы оператора Лакса в случае двух точек в голоморфной калибровке. Подставив (16) в (37) и (38), разложив полученное выражение по $\varepsilon$ и взяв предел $\varepsilon \rightarrow 0$, получим для матрицы оператора Лакса диагональную часть

$$
L^{i i}=v^{i}-\left(p^{1}\right)^{i i} E_{1}^{\prime}\left(z-x_{a}\right)
$$

и недиагональную часть

$$
L^{i j}=\left(p^{0}\right)^{i j} \varphi\left(u^{i j}, z-x_{a}\right)-\left(p^{1}\right)^{i j} \varphi^{\prime}\left(u^{i j}, z-x_{a}\right) .
$$

Матриша оператора Лакса и соответствуюшие гамильтонианы зависят от способа разложения $p^{r}$. Это означает, что можно рассматривать разложение по $\varepsilon$ и по $\bar{\varepsilon}$. В данной работе рассматривается голоморфное по $\varepsilon$ разложение $p^{r}$.

В эллиптическом случае можно заключить, что обшее пространство модулей системы в случае двух точек представляет собой расслоенное пространство с базой, определяемой параметром $\tau$, слоем которого является тор с отмеченной точкой, которой соответствует новая система с полюсом второго порядка у матришы оператора Лакса в этой точке. Это следует из рассмотрения модуля $x_{b}-x_{a}$ (при определенном $\tau$ ), т.е. мы можем зафиксировать, например, точку $x_{a}$, а $x_{b}$ будет принимать значения по всему тору, и когда $x_{b} \rightarrow x_{a}$, мы получим, что отмеченной точке соответствует новая система, полученная слиянием $x_{b}$ и $x_{a}$.

Обобшая вьшесказанное на случай слияния $r$ отмеченных точек, получим матрицу Лакса в следующем виде:

диагональная часть -

$$
L^{i i}=v^{i}+\left(p^{1}\right)^{i i} \tilde{k}_{2} E_{1}^{\prime}\left(z-x_{a}\right)+\cdots+\left(p^{r}\right)^{i i} \tilde{k}_{r} E_{1}^{(r)}\left(z-x_{a}\right),
$$

недиагональная часть -

$$
L^{i j}=\left(p^{0}\right)^{i j} \tilde{k}_{1} \varphi\left(u^{i j}, z-x_{a}\right)+\left(p^{1}\right)^{i j} \tilde{k}_{2} \varphi^{\prime}\left(u^{i j}, z-x_{a}\right)+\cdots+\left(p^{r}\right)^{i j} \tilde{k}_{r} \varphi^{(r)}\left(u^{i j}, z-x_{a}\right),
$$

где коэффициенты $\tilde{k}_{r}$ аналогичны $k_{r}$ в рациональном случае. Заметим, что симплектическая структура в этом случае совпадает с полученной в рациональном случае. Интегрирование в формуле (34), естественно, ведется по тору.

\section{5. ПРЕДЕЛ ИНОЗЕМЦЕВА}

При рассмотрении ПИ мы должны удовлетворить условию отсутствия сингулярностей в интегралах движения - следах степеней матрицы Лакса:

$$
\frac{1}{2}\left\langle L^{2}\right\rangle, \quad \frac{1}{3}\left\langle L^{3}\right\rangle, \ldots, \quad \frac{1}{k}\left\langle L^{k}\right\rangle .
$$


Как уже говорилось во введении, разлагая эти выражения по базисам функций Эйзенштейна, мы получим необходимое для интегрируемости количество гамильтонианов. Выпишем главньй неисчезающий член в функциях Эйзенштейна в пределе $\omega_{2} \rightarrow \infty$, учитывая преобразования $u=\tilde{u}+t \omega_{2}$, где $t$ - некоторый параметр.

ТАБЛИЦА 2

\begin{tabular}{|c|c|c|c|c|}
\hline функция & $t=0$ & $0<t<1$ & $t=1$ & $1<t<2$ \\
\hline$E_{1}(u)$ & $+(1 / 2) \operatorname{ch}(\tilde{u} / 2) / \operatorname{sh}(\tilde{u} / 2)$ & $+1 / 2$ & $+1 / 2$ & $+1 / 2$ \\
\hline$E_{1}^{\prime}(u)$ & $-1 / 4 \operatorname{sh}^{2}(\tilde{u} / 2)$ & $-e^{-\tilde{u}-t \omega_{2}}$ & $-2 \operatorname{ch}(\tilde{u}) e^{-\omega_{2}}$ & $-e^{\tilde{u}-(2-t) \omega_{2}}$ \\
\hline$E_{1}^{\prime \prime}(u)$ & $+(1 / 4) \operatorname{ch}(\tilde{u} / 2) / \operatorname{sh}^{3}(\tilde{u} / 2)$ & $+e^{-\tilde{u}-t \omega_{2}}$ & $-2 \operatorname{sh}(\tilde{u}) e^{-\omega_{2}}$ & $-e^{\tilde{u}-(2-t) \omega_{2}}$ \\
\hline$E_{1}^{\prime \prime \prime}(u)$ & $-1 /\left(4 \operatorname{sh}^{2}(\tilde{u} / 2)\right)-3 /\left(8 \operatorname{sh}^{4}(\tilde{u} / 2)\right)$ & $-e^{-\tilde{u}-t \omega_{2}}$ & $-2 \operatorname{ch}(\tilde{u}) e^{-\omega_{2}}$ & $-e^{\tilde{u}-(2-t) \omega_{2}}$ \\
\hline$\cdots$ & $\cdots$ & $\cdots$ & $\ldots$ & $\cdots$ \\
\hline$E_{1}^{(2 k)}(u)$ & $+(1 / 4) \operatorname{ch}(\tilde{u} / 2) / \operatorname{sh}^{3}(\tilde{u} / 2)+\cdots$ & $+e^{-\tilde{u}-t \omega_{2}}$ & $-2 \operatorname{sh}(\tilde{u}) e^{-\omega_{2}}$ & $-e^{\tilde{u}-(2-t) \omega_{2}}$ \\
\hline$E_{1}^{(2 k+1)}(u)$ & $-1 /\left(4 \operatorname{sh}^{2}(\tilde{u} / 2)\right)-\cdots$ & $-e^{-\tilde{u}-t \omega_{2}}$ & $-2 \operatorname{ch}(\tilde{u}) e^{-\omega_{2}}$ & $-e^{\tilde{u}-(2-t) \omega_{2}}$ \\
\hline
\end{tabular}

Здесь мы положили

$$
\tau=\frac{\omega_{2}}{\omega_{1}}, \quad \omega_{1}=-i \pi, \quad \operatorname{Im} \omega_{2}=0
$$

Отметим, что вырождение $E_{1}(u)$ до второго неисчезающего порядка имеет вид

$$
E_{1}(u)=\frac{1}{2} \sum_{k=-\infty}^{\infty} \operatorname{cth}\left(\frac{u}{2}-k \omega_{2}\right) \rightarrow \begin{cases}\frac{1}{2}+\frac{1}{2} e^{-\tilde{u}-t \omega_{2}}, & 0<t<1 \\ \frac{1}{2}-\operatorname{sh}(\tilde{u}) e^{-\omega_{2}}, & t=1 \\ \frac{1}{2}-\frac{1}{2} e^{\tilde{u}-(2-t) \omega_{2},} & 1<t<2\end{cases}
$$

В соответствии с этими данными будем рассматривать вырождение гамильтонианов $H^{2,2}$ и $H^{2,0}$. Сначала сделаем замечания обшего характера для всех случаев вырождения. Во-первых, при перешкалировании $E_{1}(u)=1 / 2$ при $0<t<2$. Мы не учитываем следующие члены в разложении $E_{1}(u)$, так как они заведомо исчезнут (обратное привело бы к возникновению сингулярности). Заметим, что при перешкалировании исчезают члены, содержашие $E_{1}^{\prime}(u)$ в $H^{2,2}$ и $\left(E_{1}^{\prime}(u)\right)^{2}$ в $H^{2,0}$, а также $2 v g$ в $H^{2,0}$ (из-за требования отсутствия сингулярности при перешкалировании $H^{2,4}$ ). Переменные $a, b, c, g$ и $z$ перешкалируются следующим образом:

$$
\tilde{a}=e^{-\chi_{a} \omega_{2}} a, \quad \tilde{b}=e^{-\chi_{b} \omega_{2}} b, \quad \tilde{c}=e^{-\chi_{c} \omega_{2}} c, \quad \tilde{g}=e^{-\chi_{g} \omega_{2}} g, \quad z \rightarrow \tilde{z}+s \omega_{2} .
$$

Учитывая невырожденность гамильтонианов, при перешкалированнии мы имеем следующие возможные соотношения между $t$ и некоторым параметром $s$ :

$$
s=t, 2-t .
$$


Рассмотрим случай сохранения всех скобок Пуассона между переменными $a, b, c$ и $g$ после перешкалирования. Отметим, что условия отсутствия сингулярностей в скобоках Пуассона после перешкалирования имеют вид

$$
\chi_{b} \geqslant \chi_{g}, \quad \chi_{c} \geqslant \chi_{g}, \quad \chi_{g} \geqslant \chi_{b}-\chi_{a}, \quad \chi_{g} \geqslant \chi_{c}-\chi_{a} .
$$

Знак равенства соответствует сушествованию пределов скобок Пуассона, тогда получаем

$$
\chi_{b}=\chi_{c}=\chi_{g}, \quad \chi_{a}=0 .
$$

В этом случае скобки Пуассона принимают вид

$$
\begin{array}{lll}
\{v, u\}=1, & \{\tilde{c}, \tilde{b}\}=0, \quad\{a, \tilde{g}\}=\tilde{c}-\tilde{b}, \\
\{\tilde{c}, a\}=-2 a \tilde{g}, \quad\{\tilde{b}, a\}=+2 a \tilde{g}, \quad\{\tilde{b}, \tilde{g}\}=-\frac{\tilde{b} \tilde{c}}{a}, \quad\{\tilde{c}, \tilde{g}\}=\frac{\tilde{b} \tilde{c}}{a},
\end{array}
$$

операторы Казимира -

$$
H^{2,4}=\frac{\tilde{b} \tilde{c}}{a}+\tilde{g}^{2}, \quad H^{2,3}=\tilde{b}+\tilde{c}
$$

и гамильтонианы в зависимости от $t\left(u=\tilde{u}+t \omega_{2}\right)-$

$$
H^{2,0}= \begin{cases}H^{2,2}=\frac{1}{2}(\tilde{c}-\tilde{b})+\frac{1}{2} \frac{\tilde{b}}{6} \tilde{g}^{2} e^{-\tilde{u}}+\frac{1}{12} \frac{\tilde{b} \tilde{c}}{a} e^{-\tilde{u}}, & 0<t<2, \\ v^{2}-\frac{1}{3} \tilde{g}^{2} \operatorname{ch}(\tilde{u})-(\tilde{b}-\tilde{c}) e^{\tilde{u}}+\frac{\tilde{b} \tilde{c}}{a}\left[\frac{13}{12} e^{\tilde{u}}+\frac{1}{12} e^{-\tilde{u}}\right], & t=1, \\ v^{2}-\frac{1}{6} \tilde{g}^{2} e^{\tilde{u}}-(\tilde{b}-\tilde{c}) e^{\tilde{u}}+\frac{13}{12} \frac{\tilde{b} \tilde{c}}{a} e^{\tilde{u}}, & 1<t<2 .\end{cases}
$$

\section{6. ЗАКЛЮЧЕНИЕ}

В настоящей работе были получены и описаны интегрируемые системы с полюсами порядка выше первого в матрице оператора Лакса. Открытым остается вопрос о систе$\max$, возникающих при разложении $p^{r}$ и по $\varepsilon$, и по $\bar{\varepsilon}$. Какой смысл имеют возникающие в этих системах дополнительные модули? Отметим также, что в настоящей работе при вычислении гамильтонианов новой системы в эллиптическом случае рассматривались $p \in \operatorname{sl}(2, \mathbb{C})$. Рассмотрение случая $p \in \operatorname{sl}(N, \mathbb{C})$ при $N>2$ сильно затруднено громоздкими вычислениями. Можно предположить, что в этих случаях после применения ПИ будут возникать очень близкие системы из-за одинакового вырождения функций Эйзенштейна (таблица 2).

\section{ПРИЛОЖКЕНИЕ 1}

Здесь приводятся основные сведения об эллиптических функциях, используемых в работе. Формулы заимствованы из работ [4], [10]. Определим $\vartheta$-функцию:

$\vartheta(z, \tau)=q^{1 / 8} e^{-\pi / 4}\left(e^{i \pi z}-e^{-i \pi z}\right) \prod_{n=1}^{\infty}\left(1-q^{n}\right)\left(1-q^{n} e^{2 \pi i z}\right)\left(1-q^{n} e^{-2 \pi i z}\right), \quad q=e^{2 \pi i \tau}$, 
где $\tau$ - модуль комплексного тора. Функции Эйзенштейна имеют вид

$$
\begin{gathered}
E_{1}(z, \tau)=\partial_{z} \ln \vartheta(z, \tau), \quad E_{1}(z, \tau) \approx \frac{1}{z}+\cdots \\
E_{2}(z, \tau)=-\partial_{z} E_{1}(z, \tau), \quad E_{2}(z, \tau) \approx \frac{1}{z^{2}}+\cdots \\
\frac{E_{2}^{\prime}(u)}{E_{2}(u)-E_{2}(v)}=E_{1}(u+v)+E_{1}(u-v)-2 E_{1}(u) .
\end{gathered}
$$

Их связь с функциями Вейерштрасса можно записать как

$$
\zeta(z, \tau)=E_{1}(z, \tau)+2 \eta_{1}(\tau) z, \quad \wp(z, \tau)=E_{2}(z, \tau)-2 \eta_{1}(\tau)
$$

где $\eta_{1}(\tau)=\zeta(1 / 2)$, а $\zeta(z)-\zeta$-функция Римана. Функции Эйзенштейна могут быть представлены в виде рядов

$$
\begin{gathered}
E_{1}(z)=\frac{1}{2} \sum_{k=-\infty}^{\infty} \operatorname{cth}\left(\frac{z}{2}-k \omega_{2}\right), \quad E_{2}(z)=\frac{1}{4} \sum_{k=-\infty}^{\infty} \frac{1}{\operatorname{sh}^{2}\left(z / 2-k \omega_{2}\right)} \\
E_{2}^{\prime}(z)=-\frac{1}{4} \sum_{k=-\infty}^{\infty} \frac{\operatorname{ch}\left(z / 2-k \omega_{2}\right)}{\operatorname{sh}^{3}\left(z / 2-k \omega_{2}\right)}
\end{gathered}
$$

Следуюшее выражение встречается в матрице оператора Лакса в эллиптическом случаe:

$$
\varphi(u, z)=\frac{\vartheta(u+z) \vartheta^{\prime}(0)}{\vartheta(u) \vartheta(z)}
$$

имеет полюс в точке $z=0$ и

$$
\left.\operatorname{res}\right|_{z=0} \varphi(u, z)=1
$$

Важные тождества, связанные с $\varphi$ :

$$
\varphi(u, v) \varphi(-u, v)=E_{2}(v)-E_{2}(u), \quad \varphi_{u}^{\prime}(u, v)=\varphi(u, v)\left(E_{1}(u+v)-E_{1}(u)\right)
$$

Свойства функций на решетке:

$$
\begin{aligned}
\theta(z+1) & =-\theta(z), & \theta(z+\tau) & =-q^{-1 / 2} e^{-2 \pi i z} \theta(z), \\
E_{1}(z+1) & =E_{1}(z), & E_{1}(z+\tau) & =E_{1}(z)-2 \pi i, \\
E_{2}(z+1) & =E_{2}(z), & E_{2}(z+\tau) & =E_{2}(z), \\
\varphi(u+1, z) & =\varphi(u, z), & \varphi(u+\tau, z) & =e^{-2 \pi i z} \varphi(u, z) .
\end{aligned}
$$


ПРИЛОЖЕНИЕ 2

Рассмотрим на $T^{*} G \cong \mathcal{G}^{*} \times G(G \in S L(N, \mathbb{C}))$ каноническую симплектическую формy

$$
\omega=\delta\left\langle p, g^{-1} \delta g\right\rangle
$$

где $\langle\ldots\rangle$ означает форму Киллинга, $p \in s l^{*}(N, \mathbb{C})$ и $g \in S L(N, \mathbb{C}), \delta$-оператор внешнего дифференцирования. Мы будем интересоваться пуассоновой структурой двух функций $F(p, g)$ и $H(p, g)$, определенных на $T^{*} G$. На этом многообразии сушествует скобка Ли-Пуассона [11]

$$
\{F, H\}=C_{i}^{j k} x^{i} \partial_{j} F \partial_{k} H,
$$

где $C_{i}^{j k}$ - структурные константы алгебры Ли $\mathcal{G}, \partial_{j}=\partial / \partial x^{j}$ и $x^{i}$ - координаты в фазовом пространстве.

Мы хотим придать этой записи вид, удобный для вычислений при конкретной зависимости $F$ и $H$ от $p$ и $g$. Представляя (94) в виде

$$
\{F, H\}=X_{F} H
$$

где $X_{F}$ - векторное поле, соответствующее $F$, мы можем переписать скобку Ли-Пуассона:

$$
\{F, H\}=\left\langle p,\left[\frac{\partial F}{\partial p}, \frac{\partial H}{\partial p}\right]\right\rangle-\left\langle g,\{F, H\}_{\Pi}\right\rangle,
$$

где

$$
\{F, H\}_{\Pi}=\frac{\partial F}{\partial g} \frac{\partial H}{\partial p}-\frac{\partial F}{\partial p} \frac{\partial H}{\partial g},
$$

a [, ] означает векторное произведение. Рассматривая функции $H=\left\langle p E_{j i}\right\rangle$ и $F=$ $\left\langle p E_{l k}\right\rangle$, получим

$$
\{F, H\}=p_{k j} \delta^{i l}-p_{i l} \delta^{k j}
$$

Таким образом, мы получили формулу $(7)$. В случае $p \in \operatorname{sl}(2, \mathbb{C})$ и слияния двух точек

$$
G=\left(\begin{array}{cc}
p_{1}^{0} & p_{1}^{1} \\
0 & p_{1}^{0}
\end{array}\right)
$$

Используя формулу (96), получим

$$
\begin{aligned}
& \left\{\left(p^{0}\right)^{i j},\left(p^{1}\right)^{k l}\right\}=\delta^{i l}\left(p^{1}\right)^{k, j+N}-\delta^{k j}\left(p^{1}\right)^{i, l+N}, \\
& \left\{\left(p^{0}\right)^{i j},\left(p^{0}\right)^{k l}\right\}=\frac{1}{2}\left(\delta^{i l}\left(p^{0}\right)^{k j}+\delta^{i l}\left(p^{0}\right)^{k+N, j+N}-\delta^{k j}\left(p^{0}\right)^{i l}-\delta^{k j}\left(p^{0}\right)^{i+N, l+N}\right) .
\end{aligned}
$$




\section{ПРИЛОЖЕНИЕ 3}

Рассмотрим разложение $\left\langle L^{2}\right\rangle$ в случае $2 \times 2$, т.е.

$$
L=\left(\begin{array}{ll}
L^{11} & L^{12} \\
L^{21} & L^{22}
\end{array}\right)
$$

Тогда

$$
\frac{1}{2}\left\langle L^{2}\right\rangle=\left(L^{11}\right)^{2}+L^{12} L^{21}
$$

Рассмотрим разложение диагональной и недиагональной части $\left\langle L^{2}\right\rangle$ в случае двух точек в ряд функций Эйзенштейна.

Диагональная часть. Здесь мы будем использовать свойство эллиптических функций с одинаковыми периодами отличаться на постоянное слагаемое, если они имеют одни и те же полюсы с главными частями в параллелограмме периодов:

$$
L_{i i}=\sum_{i}\left[v_{i}+\left(p_{a}\right)_{i i} E_{1}\left(z-x_{a}\right)+\left(p_{b}\right)_{i i} E_{1}\left(z-x_{b}\right)\right]
$$

Напишем предполагаемое разложение для $\left\langle L_{i i}^{2}\right\rangle$ (для удобства мы не будем писать индекс $i$, предполагая суммирование):

$$
\begin{aligned}
v^{2}+p_{a}^{2} E_{1}^{2}\left(z-x_{a}\right)+p_{b}^{2} E_{1}^{2}\left(z-x_{b}\right)+2 v p_{a} E_{1}\left(z-x_{a}\right)+2 v p_{b} E_{1}\left(z-x_{b}\right)+ \\
\quad+2 p_{a} p_{b} E_{1}\left(z-x_{a}\right) E_{1}\left(z-x_{b}\right)= \\
=-p_{a}^{2} E_{1}^{\prime}\left(z-x_{a}\right)-p_{b}^{2} E_{1}^{\prime}\left(z-x_{b}\right)+\alpha E_{1}\left(z-x_{a}\right)+\beta E_{1}\left(z-x_{b}\right)+c_{0}+v^{2},
\end{aligned}
$$

где

$$
\alpha=2 p_{a} p_{b} E_{1}\left(x_{a}-x_{b}\right)+2 v p_{a}, \quad \beta=2 p_{a} p_{b} E_{1}\left(x_{b}-x_{a}\right)+2 v p_{b} .
$$

Теперь задача состоит в определении $c_{0}$. Для этого рассмотрим $z \rightarrow x_{a}$, т.е. $z=x_{a}+\varepsilon$ при $\varepsilon \rightarrow 0$. Выразив $c_{0}$ из (103), разложив все выражения по $\varepsilon$, приведя подобные и используя условие $\left(p_{a}\right)_{i i}+\left(p_{b}\right)_{i i}=0$, получим

$$
c_{0}=+p_{a} p_{b}\left[E_{1}^{\prime}\left(x_{a}-x_{b}\right)+E_{1}^{2}\left(x_{a}-x_{b}\right)\right]
$$

Заметим, что $c_{0}$ не зависит от рассматриваемой точки (от способа вычисления).

Недиагональная часть матрицы оператора Лакса. Здесь мы будем использовать свойство эллиптических функций с одинаковыми периодами отличаться только постоянным множителем, если они имеют одинаковые нули и полюсы одной и той же кратности в параллелограмме периодов.

Вид члена от недиагональной части в $\left\langle L^{2}\right\rangle$ в случае двух точек указан в формуле (40). Встречаюшиеся там выражения от $\theta$-функций имеют вид

$$
\varphi\left(u, z-x_{a}\right) \varphi\left(-u, z-x_{a}\right), \quad \varphi\left(u, z-x_{a}\right) \varphi\left(-u, z-x_{b}\right)
$$


где

$$
\varphi\left(u^{i j}, z-x_{s}\right)=\frac{\theta\left(u^{i j}+z-x_{s}\right) \theta^{\prime}(0)}{\theta\left(u^{i j}\right) \theta\left(z-x_{s}\right)}
$$

и $s=a, b$. Нашей целью будет, анализируя нули и полюсы этих выражений, выделить эллиптическую часть (по $z-x_{s}$ ), что позволит получить разложения в ряд функций Эйзенштейна от $z-x_{s}$.

Разложение $\varphi\left(u, z-x_{a}\right) \varphi\left(-u, z-x_{a}\right)$ :

$$
\varphi\left(u, z-x_{a}\right) \varphi\left(-u, z-x_{a}\right)=\frac{\theta\left(u+z-x_{a}\right) \theta\left(-u+z-x_{a}\right)\left(\theta^{\prime}(0)\right)^{2}}{(\theta(u))^{2}\left(\theta\left(z-x_{a}\right)\right)^{2}} .
$$

Нули этого выражения

$$
u \rightarrow \pm\left(z-x_{a}\right),
$$

его полюсы

$$
\begin{aligned}
& u \rightarrow 0, \quad \varphi\left(u, z-x_{a}\right) \varphi\left(-u, z-x_{a}\right) \sim-\frac{1}{u^{2}}, \\
& z \rightarrow x_{a}, \quad \varphi\left(u, z-x_{a}\right) \varphi\left(-u, z-x_{a}\right) \sim+\frac{1}{\left(z-x_{a}\right)^{2}} .
\end{aligned}
$$

Это следует из разложения $\theta$-функций по малому параметру. Разложение будет иметь вид

$$
\varphi\left(u, z-x_{a}\right) \varphi\left(-u, z-x_{a}\right)=E_{1}^{\prime}(u)-E_{1}^{\prime}\left(z-x_{a}\right) .
$$

Аналогичное выражение мы получим и для $z-x_{b}$ :

$$
\varphi\left(u, z-x_{b}\right) \varphi\left(-u, z-x_{b}\right)=E_{1}^{\prime}(u)-E_{1}^{\prime}\left(z-x_{b}\right) .
$$

Разложение $\varphi\left(u, z-x_{a}\right) \varphi\left(-u, z-x_{b}\right):$

$$
\varphi\left(u, z-x_{a}\right) \varphi\left(-u, z-x_{b}\right)=\frac{\theta\left(u+z-x_{a}\right) \theta\left(-u+z-x_{b}\right)\left(\theta^{\prime}(0)\right)^{2}}{(\theta(u))^{2} \theta\left(z-x_{a}\right) \theta\left(z-x_{b}\right)} .
$$

Нули этого выражения

$$
u \rightarrow-\left(z-x_{a}\right), \quad u \rightarrow+\left(z-x_{b}\right)
$$

его полюсы

$$
\begin{aligned}
& u \rightarrow 0, \quad \varphi\left(u, z-x_{a}\right) \varphi\left(-u, z-x_{b}\right) \sim-\frac{1}{u^{2}}, \\
& z \rightarrow x_{a}, \quad \varphi\left(u, z-x_{a}\right) \varphi\left(-u, z-x_{b}\right) \sim+C \frac{1}{z-x_{a}}, \\
& z \rightarrow x_{b}, \quad \varphi\left(u, z-x_{a}\right) \varphi\left(-u, z-x_{b}\right) \sim-C \frac{1}{z-x_{b}} .
\end{aligned}
$$

Определим комбинацию в разложении, исходя из нулей и полюсов:

$$
\varphi\left(u, z-x_{a}\right) \varphi\left(-u, z-x_{b}\right)=C\left[-E_{1}\left(z-x_{a}\right)+E_{1}\left(z-x_{b}\right)-E_{1}(u)+E_{1}\left(u-x_{a}+x_{b}\right)\right] .
$$


Коффициент $C$ определим сравнением коэффициента в разложении левой и правой частей, например, при $z-x_{b}$ :

$$
C=\frac{\theta\left(u+x_{b}-x_{a}\right) \theta^{\prime}(0)}{\theta(u) \theta\left(x_{a}-x_{b}\right)} .
$$

Окончательно получим

$$
\begin{aligned}
\varphi\left(u, z-x_{a}\right) \varphi\left(-u, z-x_{b}\right)= & \frac{\theta\left(u+x_{b}-x_{a}\right) \theta^{\prime}(0)}{\theta(u) \theta\left(x_{a}-x_{b}\right)} \times \\
& \times\left[-E_{1}\left(z-x_{a}\right)+E_{1}\left(z-x_{b}\right)-E_{1}(u)+E_{1}\left(u-x_{a}+x_{b}\right)\right] .
\end{aligned}
$$

Аналогично для $\varphi\left(u, z-x_{b}\right) \varphi\left(-u, z-x_{a}\right)$

$$
\begin{aligned}
\varphi\left(u, z-x_{b}\right) \varphi\left(-u, z-x_{a}\right)= & \frac{\theta\left(u+x_{a}-x_{b}\right) \theta^{\prime}(0)}{\theta(u) \theta\left(x_{b}-x_{a}\right)} \times \\
& \times\left[-E_{1}\left(z-x_{b}\right)+E_{1}\left(z-x_{a}\right)-E_{1}(u)+E_{1}\left(u-x_{b}+x_{a}\right)\right] .
\end{aligned}
$$

ПРИЛОЖКЕНИЕ 4

Получим гамильтонианы новой системы. Найдем

$$
\frac{1}{2}\left\langle L^{2}\right\rangle=\left(L^{11}\right)^{2}+L^{12} L^{21}
$$

в случае системы, полученной путем слияния точек. Рассмотрим $L^{12} L^{21}$ :

$$
\begin{aligned}
L^{12} L^{21}= & {\left[\left(p^{0}\right)^{12} \varphi\left(u, z-x_{a}\right)-\left(p^{1}\right)^{12} \varphi^{\prime}\left(u, z-x_{a}\right)\right] \times } \\
& \times\left[\left(p^{0}\right)^{21} \varphi\left(-u, z-x_{a}\right)-\left(p^{1}\right)^{21} \varphi^{\prime}\left(-u, z-x_{a}\right)\right] .
\end{aligned}
$$

Чтобы вычислить это выражение, нам потребуются следующие формулы:

$$
\begin{aligned}
\varphi\left(+u, z-x_{a}\right) \varphi\left(-u, z-x_{a}\right)= & E_{1}^{\prime}(u)-E_{1}^{\prime}\left(z-x_{a}\right), \\
\varphi^{\prime}\left(+u, z-x_{a}\right) \varphi^{\prime}\left(-u, z-x_{a}\right)=- & {\left[\left(E_{1}^{\prime}(u)\right)^{2}+E_{1}^{2}(u) E_{1}^{\prime}(u)+\right.} \\
& \left.+E_{1}(u) E_{1}^{\prime \prime}(u)+\frac{1}{3} E_{1}^{\prime \prime \prime}(u)\right]+ \\
& +\left[E_{1}^{2}(u)+E_{1}^{\prime}(u)\right] E_{1}^{\prime}\left(z-x_{a}\right)-\frac{1}{6} E_{1}^{\prime \prime \prime}\left(z-x_{a}\right), \\
& +\left[E_{1}(u) E_{1}^{\prime}(u)+\frac{1}{2} E_{1}^{\prime \prime}(u)\right]+ \\
\varphi\left(+u, z-x_{a}\right) \varphi^{\prime}\left(-u, z-x_{a}\right)= & {\left[E_{1}(u) E_{1}^{\prime}\left(z-x_{a}\right)-\frac{1}{2} E_{1}^{\prime \prime}\left(z-x_{a}\right),\right.} \\
\varphi\left(-u, z-x_{a}\right) \varphi^{\prime}\left(+u, z-x_{a}\right)= & {\left[E_{1}(u) E_{1}^{\prime}(u)+\frac{1}{2} E_{1}^{\prime \prime}(u)\right]-} \\
& -E_{1}(u) E_{1}^{\prime}\left(z-x_{a}\right)-\frac{1}{2} E_{1}^{\prime \prime}\left(z-x_{a}\right) .
\end{aligned}
$$


Подставляя их в выражение для квадрата оператора Лакса, производя перегруппировку и складывая с разложением $\left\langle\left(L_{i i}\right)^{2}\right\rangle$, получим

$$
\frac{1}{2}\left\langle L^{2}\right\rangle=E_{1}^{\prime \prime \prime}\left(z-x_{a}\right) H^{2,4}+E_{1}^{\prime \prime}\left(z-x_{a}\right) H^{2,3}+E_{1}^{\prime}\left(z-x_{a}\right) H^{2,2}+H^{2,0},
$$

где

$$
\begin{aligned}
H^{2,4}= & -\frac{1}{6}\left(p^{1}\right)^{12}\left(p^{1}\right)^{21}-\frac{1}{6}\left(p^{1}\right)^{11}\left(p^{1}\right)^{11}, \quad H^{2,3}=\frac{1}{2}\left[\left(p^{0}\right)^{12}\left(p^{1}\right)^{21}+\left(p^{0}\right)^{21}\left(p^{1}\right)^{12}\right], \\
H^{2,2}= & -\left(p^{0}\right)^{12}\left(p^{0}\right)^{21}+E_{1}(u)\left[-\left(p^{0}\right)^{12}\left(p^{1}\right)^{21}+\left(p^{0}\right)^{21}\left(p^{1}\right)^{12}\right]+ \\
& +\left(p^{1}\right)^{12}\left(p^{1}\right)^{21}\left[E_{1}^{\prime}(u)+E_{1}^{2}(u)\right]-2 v\left(p^{1}\right)^{11} \\
H^{2,0}= & v^{2}+\left(\left(p^{1}\right)^{11}\right)^{2}\left[\frac{1}{6} E_{1}^{\prime \prime \prime}(u)+\left(E_{1}^{\prime}(u)\right)^{2}\right]+\left(p^{0}\right)^{12}\left(p^{0}\right)^{21} E_{1}^{\prime}(u)+ \\
& +\left[\left(p^{0}\right)^{12}\left(p^{1}\right)^{21}-\left(p^{0}\right)^{21}\left(p^{1}\right)^{12}\right]\left[E_{1}(u) E_{1}^{\prime}(u)+\frac{1}{2} E_{1}^{\prime \prime}(u)\right]- \\
& -\left(p^{1}\right)^{12}\left(p^{1}\right)^{21}\left[\left(E_{1}^{\prime}(u)\right)^{2}+E_{1}^{2}(u) E_{1}^{\prime}(u)+E_{1}(u) E_{1}^{\prime \prime}(u)+\frac{1}{3} E_{1}^{\prime \prime \prime}(u)\right]
\end{aligned}
$$

Благодарности. Автор выражает благодарность В. Долотину, А. Зотову, А. Миронову, Д. Талалаеву и А. Червову за полезные замечания, А. Левину за многочисленные обсуждения. Особую признательность автор хочет выразить М. Ольшанецкому за предложенную тему, поддержку и внимание в процессе написания статьи. Работа была выполнена при частичной поддержке гранта РФФИ № 00-02-16530 и гранта поддержки научных школ № 00-15-96557.

\section{Список литературы}

[1] M. Gaudin. J. Physique. 1976. V. 37. P. 1087.

[2] N. Nekrasov. Commun. Math. Phys. 1996. V. 180. P. 587; hep-th/9503157.

[3] B. Enriques, V. Rubtsov. Math. Res. Lett. 1996. V. 3. P. 343.

[4] A. M. Levin, M. A. Olshanetsky. Hierarchies of isomonodromic deformations and Hitchin systems. hep-th/9709207.

[5] N. Hitchin. Duke Math. J. 1987. V. 54. № 1. P. 91.

[6] A. Beauville. Acta Math. 1990. V. 164. № 3/4. P. 211.

[7] A. Chervov, D. Talalaev. Hitchin systems on singular curves. II. Gluing subschemes. hep-th/ 0309059 .

[8] А. В. Зотов, Ю. Б. Черняков. ТМФ. 2001. Т. 129. № 2. С. 258.

[9] V. I. Inozemtsev. Commun. Math. Phys. 1989. V. 121. P. 629.

[10] E. D'Hoker, D. H. Phong. Lectures on supersymmetric Yang-Mills theory and integrable systems. hep-th/9912271.

[11] А. М. Переломов. Интегрируемые системы классической механики и алгебры Ли. М.: Наука, 1990. 